\title{
Article \\ In-Depth Verification of a Numerical Model for an Axisymmetric RC Dome
}

\author{
Przemysław Czumaj (D), Sławomir Dudziak*(D) and Zbigniew Kacprzyk (D) \\ Institute of Building Engineering, Faculty of Civil Engineering, Warsaw University of Technology, \\ Al. Armii Ludowej 16, 00-637 Warsaw, Poland; p.czumaj@il.pw.edu.pl (P.C.); z.kacprzyk@il.pw.edu.pl (Z.K.) \\ * Correspondence: s.dudziak@il.pw.edu.pl
}

\begin{abstract}
The designers of civil engineering structures often have to face the problem of the reliability of complex computational analyses performed most often with the Finite Element Method (FEM). Any assessment of reliability of such analyses is difficult and can only be approximate. The present paper puts forward a new method of verification and validation of the structural analyses upon an illustrative example of a dome strengthened by circumferential ribs along the upper and lower edges. Four computational systems were used, namely Abaqus, Autodesk Robot, Dlubal RFEM, and FEAS. Different models were also analyzed-two-dimensional (2D) and three-dimensional (3D) ones using continuum, bar, and shell finite elements. The results of the static (with two kinds of load-self-weight and load distributed along the upper ring) and modal analyses are presented. A detailed comparison between the systems' and models' predictions was made. In general, the spatial models predicted a less stiff behavior of the analyzed dome than the planar models. The good agreement between different models and systems was obtained for the first natural frequency with axisymmetric eigenmodes (except from the Autodesk Robot system). The presented approach to the verification of complex shell-bar models can be effectively applied by structural designers.
\end{abstract}

Keywords: FEM; dome; modal analysis; reliability of calculations; verification and validation

Kacprzyk, Z. In-Depth Verification of

a Numerical Model for an

Axisymmetric RC Dome. Symmetry

2021, 13, 2152. https://doi.org/

$10.3390 /$ sym 13112152

Academic Editors: Yeran Sun,

Tomasz Lewiński, Shaohua Wang and Ting On Chan

Received: 12 October 2021

Accepted: 5 November 2021

Published: 11 November 2021

Publisher's Note: MDPI stays neutral with regard to jurisdictional claims in published maps and institutional affiliations.

\section{Introduction}

The Finite Element Method (FEM) has become a universal tool of the analysis of the response of engineering structures. Many FEM-based computational systems are used in practice. However, not all software companies provide theory manuals for their products: the users have to use the software without being informed on how the finite element is constructed; in some cases, the software serves as a black box. Thus, the process of the validation and verification of the computational models acquires particular significance [1]. In general, the validation process makes it possible to answer the question: "Are we solving the proper mathematical equations modeling the considered mechanical phenomena?". On the other hand, the verification process means answering the question: "Are we solving these equations properly?". Thus, the validation concerns the physics, while the verification refers to the problem of the correctness of the assumed approximations [2]. These assessments are especially difficult in the case of large-scale problems of the mechanics of structures composed of bars, plates, and shells. The validation requires experimental evidence; hence, it cannot be performed before the structure is erected. On the other hand, the mathematical analysis is vague, due to lack of theorems on the convergence of FEM schemes applied to complicated multifold shell-like structures with jumps (so-called offsets) of the reference surfaces along the edges, leading to the eccentricity of the resulting membrane forces [3]. The present paper shows how to deal with such problems by concentrating the attention on the stress analysis of a dome reinforced by circumferential ribs along both edges.

Due to their small mass and high stiffness, rotationally symmetric domes are frequently designed, treated also as attractive architectural forms [4]; hence, the literature 
devoted to them is rich, including monographs and in-depth studies. In the past, the stress analyses of domes were performed analytically; hence, the analyses were affected by strong assumptions of the shell theories, concerning, e.g., the methods of modeling the boundary conditions and the loads, keeping the rotationally symmetric property of the problem $[5,6]$. Currently, due to the limitations of the analytical approach, FEM analysis is usually used; see [7-10]. In some studies, the quasi-brittle behavior of the materials from which the domes are constructed is also taken into account; cf. [11,12]. Domes are characterized by a very advantageous area-to-volume ratio, hence being attractive from the point of view of sustainability; this is why their thermal properties are the subject of intensive studies $[13,14]$.

The papers dealing with detailed comparison of the results obtained with different FE codes are rather scarce. Except the previous papers published by the authors, e.g., [15] concerning a ribbed floor or $[16,17]$ regarding a cylindrical tank on a deformable subsoil, the paper [18] on the detailed validation of the numerical model of a bridge using three different FE codes (Robot, SOFiSTiK, and Abaqus) and different modeling techniques can be mentioned. The comparison of different numerical models of the nonlinear behavior of reinforced concrete was presented in [19]. However, a dome with ring ribs has not been considered in any of these papers.

This paper puts forward the extensive process of the verification and validation of an illustrative example of a constructed dome with ribs along the edges, whose geometry is depicted in Figure 1. The global coordinate systems in 2D and 3D models are also shown. Due to the discontinuous support conditions, the problem is not rotationally symmetric; see Figure 2. The dome was modeled using various FEM systems. The three-dimensional FEM analysis was confronted with the approximate rotationally symmetric modeling, neglecting the influence of the discontinuity of the support along the lower edge. The rotationally symmetric FEM model is viewed as being referential for the FE modeling, which is based on connecting the shell elements with the finite elements modeling the circumferential ribs. In the sequel, the bar-shell models are discussed, making it possible to give up the assumption of rotational symmetry and assume much more realistic boundary conditions. Thus, we considered three models with approximate treatment of the support conditions and a fourth model in which these conditions are properly taken into account. It is worth mentioning that the analyzed dome cannot be considered as a shallow one: the radius of the spherical part is only $6 \mathrm{~m}$. Consequently, it is difficult to obtain reliable results with classical simplified analytical methods (as the variants of force method presented in [5,6]; the examples there concerned a sphere of $25 \mathrm{~m}$ in radius).

The following four FE systems are used: Autodesk Robot [20], RFEM [21], Abaqus [22], and the author's system FEAS [23]. The first two systems include the modules for the dimensioning of the structures using the common relevant standards. Abaqus makes it possible to incorporate nonlinear effects, both geometrical and material. The static and modal analyses were performed; the natural frequencies were computed; the corresponding vibration modes were constructed.

The paper aims to place strong emphasis on the necessity of performing the validation and verification of FEM-based results, the conclusions being directed to civil engineers. Our warnings concern bar-shell structures used as dome roofs of buildings. The analyses of such structures should not only be thoroughly checked, but the correctness of the FE results should not be taken for granted either. 

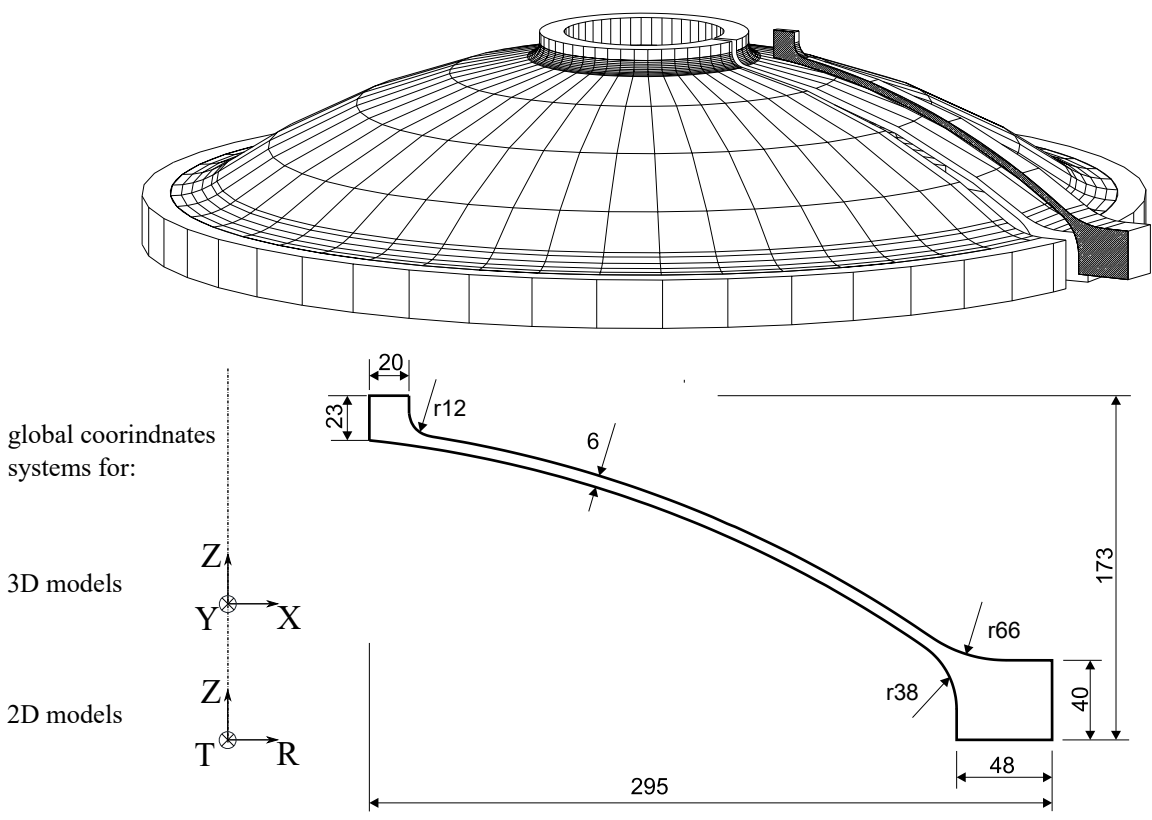

Figure 1. An analyzed dome: an axonometric view and the cross-section with the global coordinate systems. The dimensions are given in centimeters.

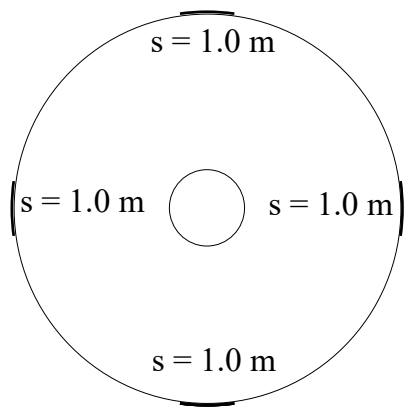

Figure 2. A projection of the shell with supports marked: the length of a single support zone is $1.0 \mathrm{~m}$.

\section{Computational Models}

2.1. Main Assumptions

Four computational models were analyzed:

p1 A two-dimensional model with axisymmetric continuum finite elements;

p2 A two-dimensional model with axisymmetric shell finite elements and ring beam elements;

p3 A three-dimensional model with shell and bar elements with continuous support of the structure;

p4 A three-dimensional model with shell and bar elements with discontinuous support of the structure (the boundary conditions corresponding to the real situation).

In each model, the linear and isotropic material model was assumed with parameters corresponding to heavily reinforced concrete:

- $\quad E=27,000 \mathrm{MPa}-$ Young's modulus;

- $v=\frac{1}{6}$-Poisson's ratio;

- $\quad \varrho=2800 \mathrm{~kg} / \mathrm{m}^{3}$ — the bulk density.

For each model, a static analysis was performed with two load cases:

a. Self-weight load;

b. Evenly distributed load on the top ring beam with an intensity of $500 \mathrm{kN} / \mathrm{m}$ (the resultant force acting on the top ring with a radius of $r=0.751 \mathrm{~m}$ is $2 \pi r=2359.34 \mathrm{kN}$ ). 
A modal analysis was also performed.

In the modal analysis, in addition to the stiffness matrix, the mass matrix has to be determined. The computational systems allow for assuming mass in various ways: one can assume a distributed mass, mass concentrated in nodes with rotations, and mass concentrated in nodes without rotation. In the examples below, we took the distributed mass and built a consistent mass matrix. In the calculations, we determined the first 10 natural frequencies. Solving the eigenvalue problem with matrices of big dimensions is a difficult numerical task, and computational systems use different procedures. The most popular are the Lanczos method and subspace iteration, cf. [24,25]. We used both of these methods in the calculations.

To compare the results of the static analysis, the vertical displacement of the axis of the upper ring was adopted and, in the case of the modal analysis, the eigenfrequencies and the eigenmodes corresponding to them.

A very important stage of modeling is the approximation of the structure's geometry. In the $\mathbf{p} \mathbf{1}$ model, the geometry can be mapped very accurately, whereas in the $\mathbf{p} \mathbf{2}, \mathbf{p} 3$, and p4 models, the three-dimensional object is approximated by surfaces and axes (marking the center of gravity of the rings). One can approximate the geometry with surfaces and axes in a variety of ways. Figure 3 shows examples of methods of modeling geometry. In the computational model shown in Figure 3b, we assumed the surface of the shell on the upper surface of the dome and extended the span of the surface to the centers of gravity of the rings (approximately); see Figure 3a. In the computational model shown in Figure 3d, the surface of the shell was assumed in the middle of the dome's thickness and the span of the surface was extended to the centers of gravity of the rims (approximately); see Figure 3c. On the other hand, in the model from Figure 3f, the shell surface was assumed to be in the middle of the dome's thickness and the span of the shell was not increased. The axes of the rings were located in the centers of gravity of the sections, and in this computational model, we assumed the offset of the bars' axes.

(a)

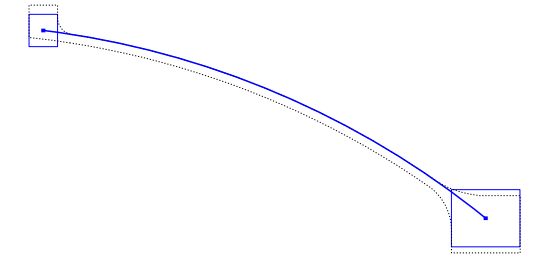

(c)
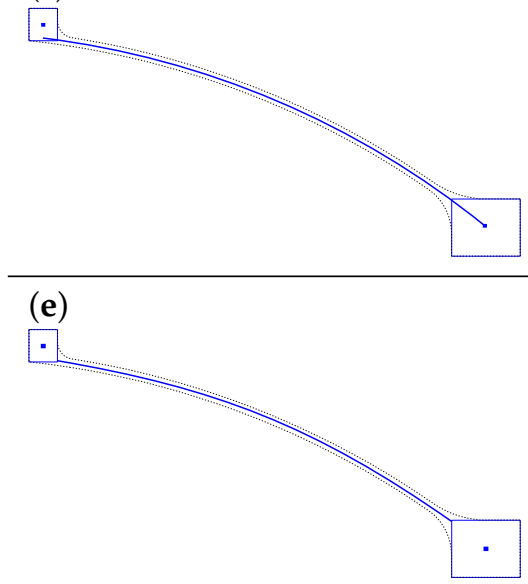

(b)

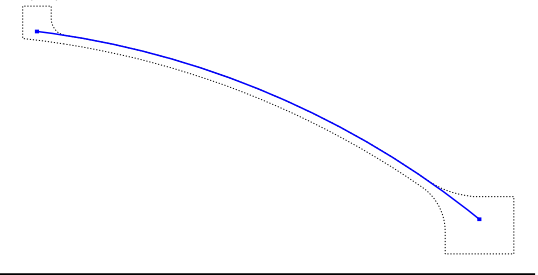

(d)

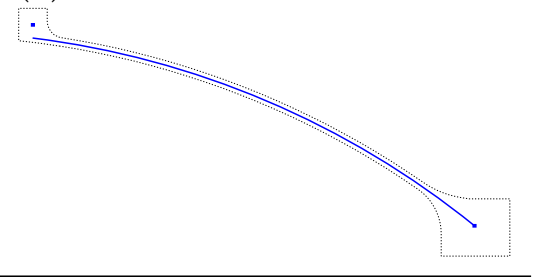

(f)

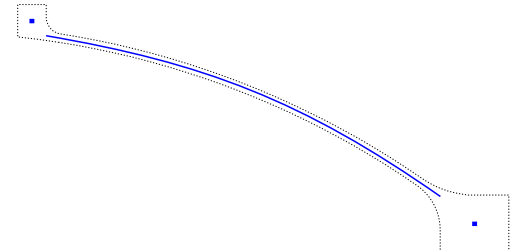

Figure 3. Modeling of the shell: (a) alignment to the top surface of the shell, (b) calculation model, (c) alignment to the middle surface of the shell, (d) calculation model, (e) alignment to the middle surface of the shell with the trim of the shell along the beam contour, and (f) the computational model. 
The scheme shown in Figure 3b was used in the p2, p3, and p4 models. The computational model shown in Figure $3 \mathrm{f}$ was used only in the Abaqus system in the $\mathbf{p} 3$ and $\mathbf{p} 4$ models. The $\mathbf{p} 1$ model was assumed as the reference for the $\mathbf{p} 2$ and $\mathbf{p} 3$ models.

\subsection{Description of the Models}

\subsubsection{Model p1: The Theory of Elasticity in the Axisymmetric State}

The calculations of the rotational symmetric solid were performed with three FE systems: FEAS, ARSAP, and Abaqus. It was assumed that the supports of the bottom ring were located at one point (more precisely, along a circle) in the center of the bottom surface of the ring, which ensures geometrical invariance in the case of the rotational symmetry. Thus, we blocked only one degree of freedom: vertical displacement $\left(u_{Z}=0\right)$. In each of the systems, at least two finite element meshes were used, observing the convergence of the problem. The FEAS system uses triangular three-node finite elements, whereas ARSAP employs three- and four-node elements because of the applied meshing technique. In the Abaqus system, a six-node triangular element (AX6 according to the program documentation) is used to obtain the most accurate results. Such elements are not available in the other two FE codes.

Figure 4 shows the FEM meshes from different systems prepared for the calculations. The dimensions of the model after discretization (the number of finite elements and nodes) are given below. In the ARSAP system, in curvilinear structures, the element generator is related to the geometry description. A detailed description of the geometry requires generating a large number of elements; see Figure 4 . In the given example, the division into elements can be considered as very dense.

(a)

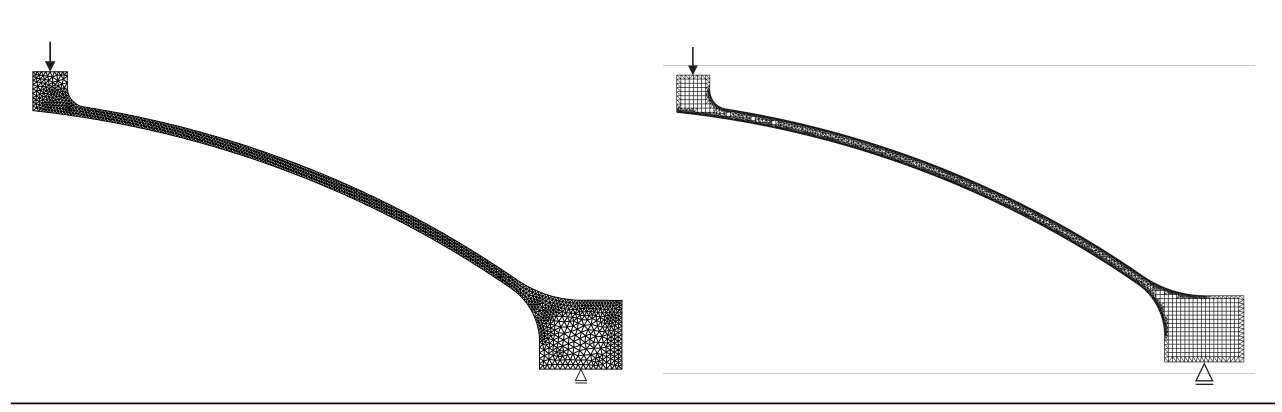

(c)

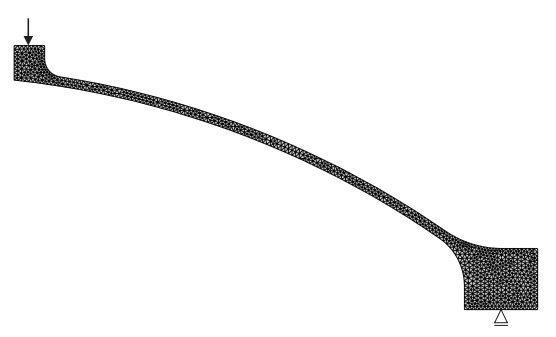

(b)

Figure 4. FE meshes adopted in the p1 model analysis: (a) FEAS-division into elements: 1482 nodes, 2486 elements; (b) ARSAP_division into elements: 3345 nodes, 4974 elements; (c) Abaqus—division into elements: 3462 nodes, 1571 elements

\subsubsection{Model p2: 2D Shell of Revolution with Ring Beams}

This section describes the model of the dome, assuming the model of a rotationally symmetrical shell with ring beams. Ring elements are uncommon in computing systems; 
hence, the calculations were performed only in the Abaqus and FEAS systems. In the Abaqus system, we used 17 3-node SAX2 shell elements ( 35 nodes in total). The ring beams were considered as two-parameter springs with horizontal rotational and translational stiffness. The spring stiffness was calculated on the basis of the work [5], neglecting the eccentricities in the connections of the shell with the rings (see Figure 3 ). We calculated the translational $\left(k_{u}\right)$ and rotational $\left(k_{\varphi}\right)$ stiffnesses according to the following formulas [5]:

$$
\begin{aligned}
& k_{u}=\frac{E A}{R^{2}} \\
& k_{\varphi}=\frac{E J}{R^{2}} .
\end{aligned}
$$

The FEAS system adopts 120 nodes and 119 flat axisymmetric shell elements and two single-node ring elements (top and bottom ring beams), [26]. The preliminary analysis of convergence showed that the division of the structure into 59 shell elements (60 nodes) and 2 ring elements did not guarantee correct results. As in the previous model, only one kinematic boundary condition was assumed: $u_{Z}=0$ at the center of the bottom ring.

\subsubsection{Model p3: 3D Shell-Bar Model}

In the p3 model, the dome is modeled with three-dimensional shell and bar elements. We did not use the rotational symmetry of the task in this model. Computational models were prepared in all four FEM systems. In each system, FE meshes of different densities were generated, and the results were converged. The list of the analyzed models is shown in Table 1.

Table 1. Summary of the presented calculation models.

\begin{tabular}{ccccc}
\hline Model & Shell Element & Bar Element & Coaxiality of Ring Bars & System \\
\hline p3 & 8-node & 3-node & no, Figure 3f & Abaqus \\
offset & curved & curved & & \\
p3 & 8-node & 3-node & yes, Figure 3b & Abaqus \\
& curved & curved & & \\
p3 & 3-node flat & 2-node & yes, Figure 3b & FEAS \\
p3 & 4-node flat & 2-node & yes, Figure 3b & ARSAP \\
p3 & 4-node & 2-node & yes, Figure 3b & RFEM \\
\hline
\end{tabular}

The analyzed models together with information on the dimensions of discrete models are presented in Figure 5. In the Abaqus system, models using curved eight-node shell elements (symbol S8R) and three-node bar elements (symbol B32) were analyzed. In the FEAS system, a model was made using three-node shell elements and two-node bar elements. The division into finite elements was obtained using a generator for geometrical primitives. Such a generator allows for regular division into finite elements according to the given parameters; see [23]. In the ARSAP system, the model was prepared using flat four-node shell elements and two-node bar elements. A similar model was developed in the RFEM code. Boundary conditions were assumed by blocking vertical displacements in the nodes of the bottom ring $u_{Z}=0$. In order to ensure the geometric invariance of the system in one node, translational displacements in the other two directions and rotation around the vertical axis were additionally restrained $\left(u_{X}=u_{Y}=0\right)$. In the absence of such an assumption, the system was geometrically variable, which resulted in a singular stiffness matrix, and as a consequence numerical problems. 
(a)

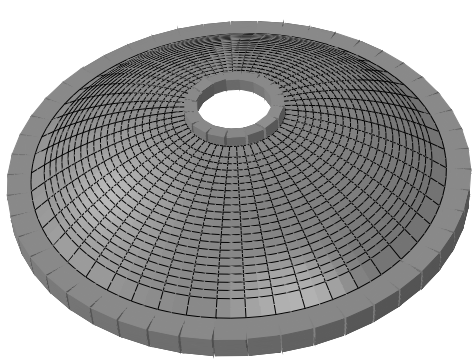

(c)

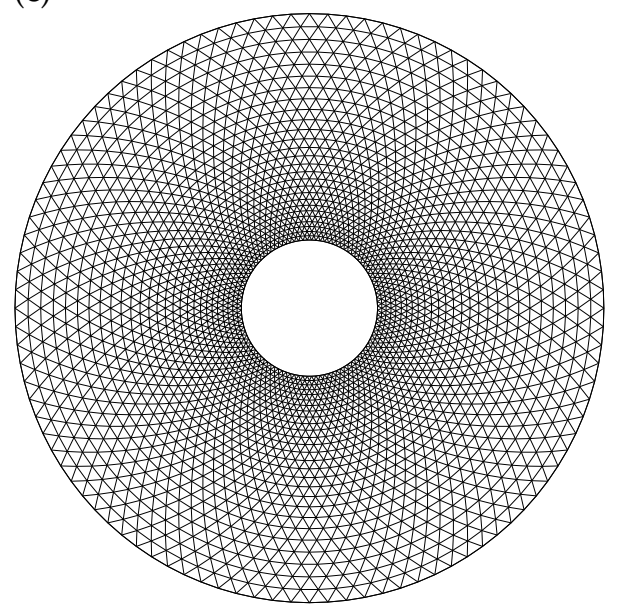

(b)

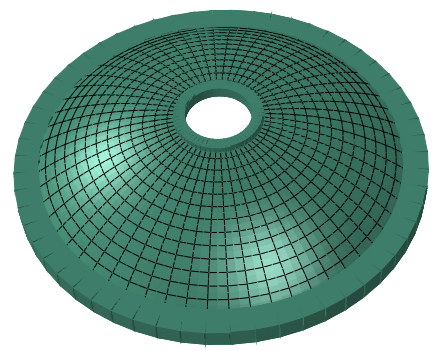

(d)

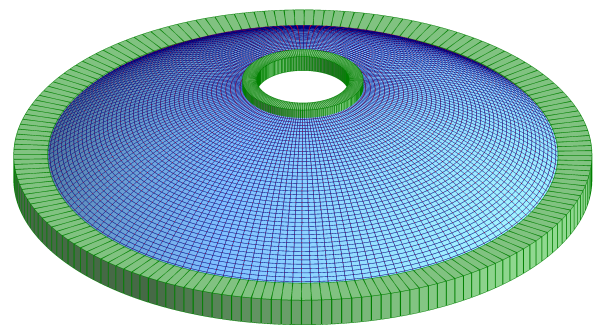

(e)

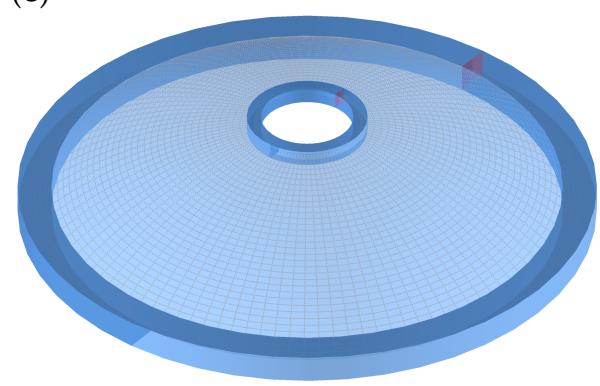

Figure 5. Model p3: (a) Abaqus—computational model p3 offset, nodes: 3312, shell elements: 1008, bar elements: 64, (b) Abaqus—computational model p3, nodes: 2900, shell elements: 812, bar elements: 116; (c) FEAS — computational model p3 (plan view) - 2800: nodes, shell elements: 5400, members: 200; (d) ARSAP_computational model p3, nodes: 9190, shell elements: 3836, members: 360; (e) RFEM—computational model p3, nodes: 3780, shell elements: 3672, bar elements: 216.

\subsubsection{Model p4: 3D Shell-Bar Model with Discontinuous Supports}

Finally, the target dome model was analyzed. The dome was supported with sections along the bottom ring (the length of a single support section was $1 \mathrm{~m}$ ); see Figure 2. Such segmental support is often found in objects with partially supported domes, sometimes such that the supports are called "sails". The computational models were the same as for the p 3 model, except for the boundary conditions. At the nodes located on the support sections, all translational degrees of freedom were restrained: $u_{X}=u_{Y}=u_{Z}=0$.

\section{Results of the Analyses}

\subsection{Static Analysis}

Taking into account the self-weight in the FE systems can be done automatically: the user only enters the material's density. The value of the gravity force was calculated 
based on the geometry of the model. Examples of the results of the vertical reactions and displacements of the upper ring due to the self-weight for the $\mathbf{p} \mathbf{1}$ and $\mathbf{p} 3$ models are presented in Tables 2 and 3, respectively. It is worth comparing the reaction values. The differences in the reaction values resulted from the accuracy of the geometry mapping (and this is mainly a feature of the structure description; the geometry mapping differs in individual models). As for such a detailed description of the structure in the computational systems, the differences can be considered quite large.

Table 2. Summary of displacements and reactions: p1 model, self-weight.

\begin{tabular}{ccc}
\hline & $\boldsymbol{u}_{\boldsymbol{z}}(\mathbf{m}) \cdot \mathbf{1 0 ^ { - \mathbf { 5 } }}$ & $\boldsymbol{R}_{\boldsymbol{z}}(\mathbf{k N})$ \\
\hline Abaqus & -5.05 & 214.00 \\
FEAS & -5.32 & 211.42 \\
ARSAP & -5.34 & 211.02 \\
\hline
\end{tabular}

Table 3. Summary of displacements and reactions: p3 model, own weight.

\begin{tabular}{cccc}
\hline Model & System & $\boldsymbol{u}_{\boldsymbol{z}}(\mathbf{m}) \cdot \mathbf{\cdot 1 0 ^ { - 5 }}$ & $\boldsymbol{R}_{\boldsymbol{z}} \mathbf{( k N )}$ \\
\hline p3 & Abaqus & -5.39 & 202.81 \\
offset & & & \\
p3 & Abaqus & -5.75 & 220.40 \\
p3 & FEAS & -5.43 & 202.68 \\
p3 & ARSAP & -5.64 & 216.60 \\
p3 & RFEM & -5.64 & 216.49 \\
\hline
\end{tabular}

The deformation of the structure obtained with the use of the $\mathbf{p} \mathbf{1}$ model caused by the self-weight is presented in Figure 6. In Figure 7, the state of displacement for the same model can be found due to the load on the top ring. Figure 8 shows the map of the vertical displacements of the shell due to self-weight obtained with the use of the Abaqus system for the p3 model, whereas Figure 9 shows the deformation of the dome under the upper ring's load obtained with the FEAS system.

(a)

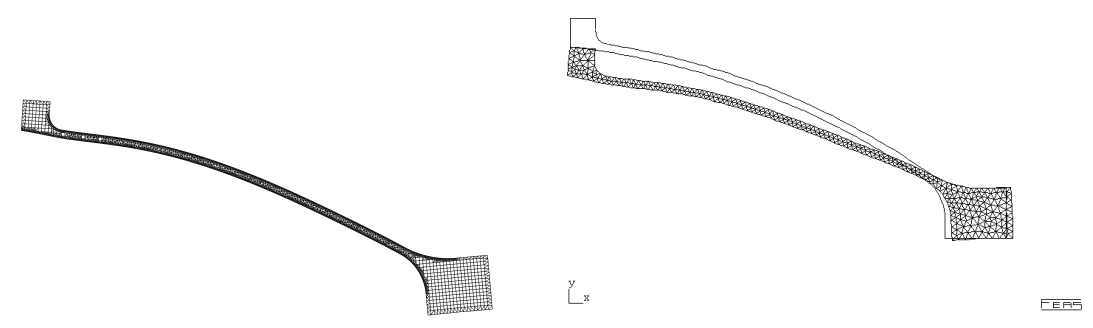

(b)

Figure 6. Deformation from self-weight: (a) ARSAP; (b) FEAS.

In Table 4, the vertical displacements of the models under self-weight load are summarized, while in Table 5, the displacements under the load of the upper ring can be found. In the case of models that were analyzed in at least three systems, the mean, the Standard Deviation (STD), and the Coefficient of Variation (CoV) were also calculated, allowing for the evaluation of the dispersion of the results obtained with the use of different systems. The quotients of the calculated displacements and the reference value are given in brackets, which were taken as the mean for the $\mathbf{p} 1$ models (in bold in the table). 
(a)

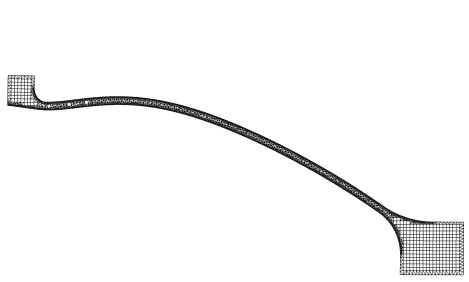

(b)

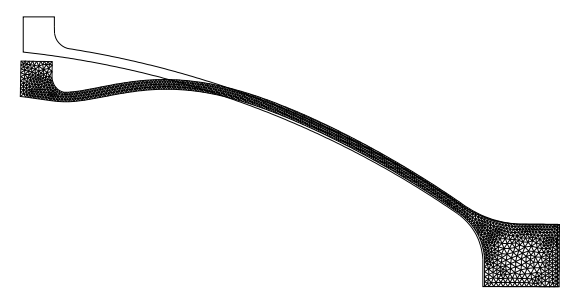

Figure 7. Deformation under the load on the top ring: (a) ARSAP; (b) FEAS.
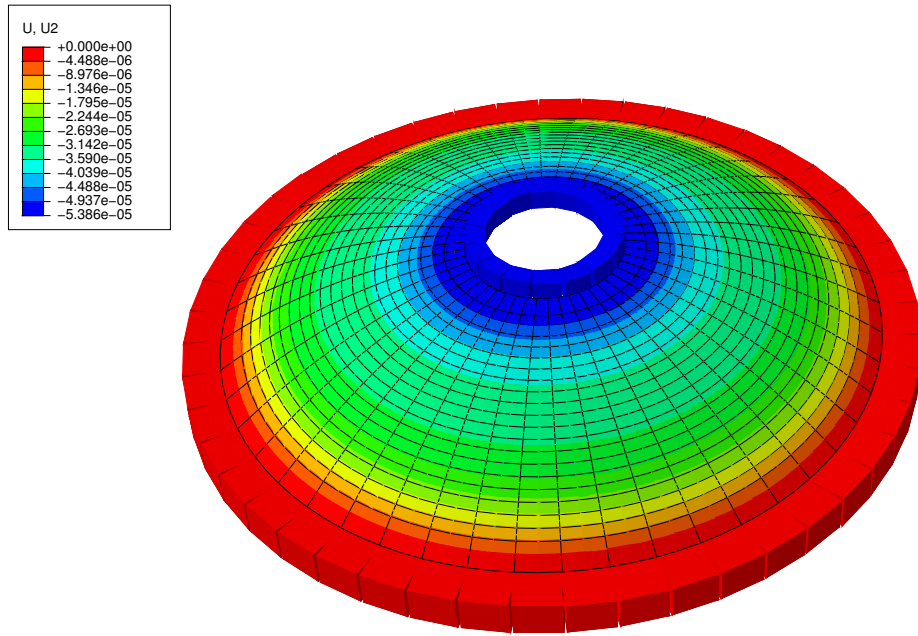

Figure 8. Deformation under the self-weight: Abaqus.

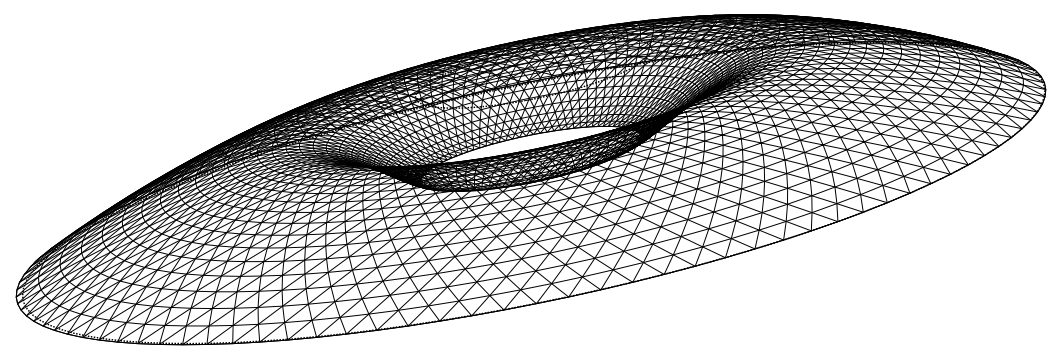

Figure 9. Deformation under the load on the top ring: FEAS.

Table 4. Summary of displacements $u_{z}(\mathrm{~m}) \cdot 10^{-5}$ : self-weight.

\begin{tabular}{|c|c|c|c|c|c|c|c|c|c|}
\hline System & p1 & p2 & & p3 & & p3 Offset & $\mathrm{p} 4$ & & p4 Offset \\
\hline Abaqus & 5.05 & 5.75 & (1.10) & 5.75 & (1.10) & (1.03) & 7.55 & (1.44) & $(1.30)$ \\
\hline FEAS & 5.32 & 5.37 & (1.03) & 5.43 & (1.04) & & 8.61 & (1.64) & \\
\hline ARSAP & 5.34 & & & 5.64 & (1.08) & & 9.57 & (1.83) & \\
\hline Dlubal & & & & 5.64 & (1.08) & & 8.48 & (1.62) & \\
\hline mean & 5.24 & & & 5.62 & (1.07) & & 8.55 & (1.63) & \\
\hline STD & 0.13 & & & 0.12 & & & 0.72 & & \\
\hline $\mathrm{CoV}$ & 0.03 & & & 0.02 & & & 0.08 & & \\
\hline
\end{tabular}


Table 5. Summary of displacements $u_{z}(\mathrm{~cm})$ : load on the upper ring.

\begin{tabular}{|c|c|c|c|c|c|c|c|c|c|}
\hline System & p1 & p2 & & p3 & & p3 Offset & $\mathrm{p} 4$ & & p4 Offset \\
\hline Abaqus & 0.97 & 1.27 & (1.33) & 1.27 & (1.33) & (1.11) & 1.26 & (1.32) & $(1.10)$ \\
\hline FEAS & 0.95 & 1.01 & (1.06) & 1.15 & (1.20) & & 1.22 & (1.28) & \\
\hline ARSAP & 0.95 & & & 1.30 & (1.36) & & 1.29 & (1.35) & \\
\hline Dlubal & & & & 1.30 & (1.36) & & 1.30 & (1.36) & \\
\hline mean & 0.96 & & & 1.26 & (1.31) & & 1.27 & (1.33) & \\
\hline STD & 0.01 & & & 0.06 & & & 0.03 & & \\
\hline $\mathrm{CoV}$ & 0.01 & & & 0.05 & & & 0.02 & & \\
\hline
\end{tabular}

\subsection{Modal Analysis}

This section presents the results of the modal analysis of the models used earlier in the static case. The values of the natural frequencies for the $\mathbf{p} \mathbf{1}$ model are summarized in Table 6, and the corresponding modes of free vibration are shown in Figure 10. It is worth paying attention to the values obtained from the ARSAP system, which differ significantly from the other results. In the first phase of the calculations, the authors were convinced that the error was due to badly prepared and entered data. No error was found after extensive data verification. In order to verify the results, two more calculation models, p1, in the ARSAP system were prepared (see Table 7) with a different division into finite elements and reprepared geometry. We also performed calculations for various inertia matrices (distributed and concentrated mass), for triangular and quadrilateral elements. The obtained results were similar to each other. Unfortunately, the verification of the model indicated that the eigenvalues for the rotationally symmetric model in the ARSAP system were calculated incorrectly.

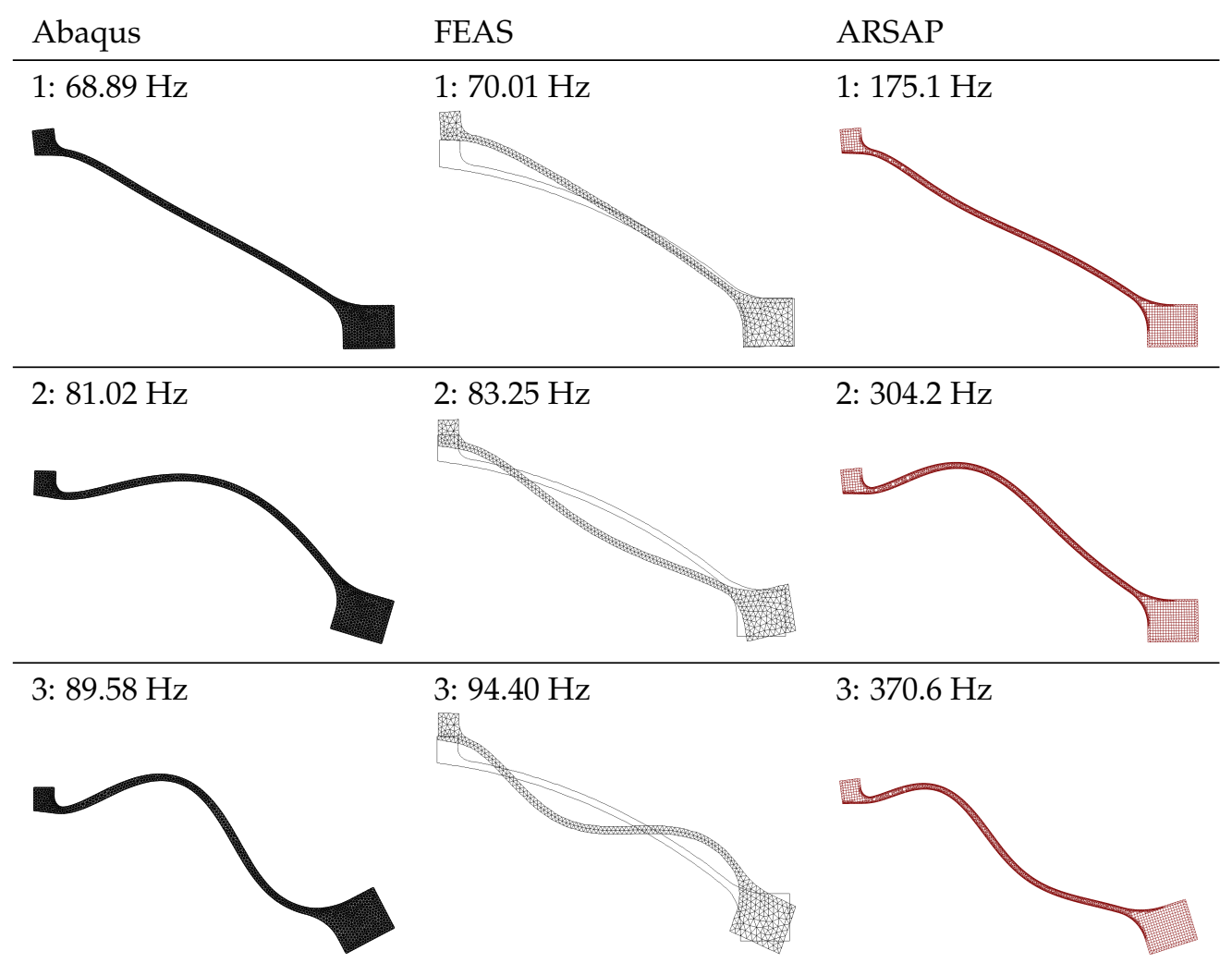

Figure 10. Model p1: frequencies and modes of natural vibration. 
Table 6. List of the first natural frequencies of the dome for the p1 model (Hz).

\begin{tabular}{cccccc}
\hline System & $f_{1}$ & $f_{2}$ & $f_{3}$ & $f_{4}$ & $f_{5}$ \\
\hline Abaqus & 68.89 & 81.02 & 89.58 & 114.7 & 160.8 \\
FEAS & 69.54 & 81.34 & 90.65 & 117.7 & 166.7 \\
ARSAP & 171.5 & 304.2 & 370.6 & 444.5 & 593.9 \\
\hline
\end{tabular}

Table 7. List of the first natural frequencies of the dome for the $\mathbf{p} \mathbf{1}$ model $(\mathrm{Hz})$ calculated in the ARSAP system.

\begin{tabular}{cccccc}
\hline System & $f_{\mathbf{1}}$ & $f_{\mathbf{2}}$ & $f_{3}$ & $f_{\mathbf{4}}$ & $f_{\mathbf{5}}$ \\
\hline ARSAP (379 nodes) & 175.0 & 309.5 & 378.1 & 470.6 & 642.2 \\
ARSAP (940 nodes) & 172.1 & 305.0 & 371.7 & 451.0 & 608.1 \\
ARSAP (3345 nodes) & 171.5 & 304.2 & 370.6 & 444.5 & 593.9 \\
\hline
\end{tabular}

Table 8 lists the natural frequencies for the $\mathbf{p} 2$ model. The first two zero values were obtained in the Abaqus system. Therefore, in Table 8, the next number of the eigenvalue is given in brackets. Figure 11 shows the first three modes of free vibration. The results from the Abaqus and FEAS systems obtained in the $\mathbf{p} 2$ model were similar to the values from the $\mathbf{p} 1$ model.

Table 8. List of the first natural frequencies of the dome for the $\mathbf{p} 2(\mathrm{~Hz})$ model.

\begin{tabular}{cccccc}
\hline System & $f_{1}$ & $f_{2}$ & $f_{3}$ & $f_{4}$ & $f_{5}$ \\
\hline Abaqus & $68.53(3)$ & $79.57(4)$ & $91.97(5)$ & $118.7(6)$ & $163.57(7)$ \\
FEAS & 68.61 & 82.11 & 96.72 & 116.8 & 144.1 \\
\hline
\end{tabular}

In Table 9, the natural frequencies for the p3 model are compiled. The eigenvalue number is given in brackets. For the Abaqus system, we included the results for the $\mathbf{p} 3$ and $\mathbf{p} 3$ offset models; cf. Table 1. In the Abaqus system, it is possible to find a solution to the eigenvalue problem without introducing a point block, with a singular stiffness matrix. Due to the possible three rigid movements, three zero-natural frequencies were obtained, which were not included in the summary. Due to the rotational symmetry of the problem, there were also double natural frequencies, which are not repeated in the tables. Therefore, the numbers of the natural frequencies are placed in brackets.

Table 9. List of the first natural frequencies of the dome for the $\mathrm{p} 3(\mathrm{~Hz})$ model.

\begin{tabular}{ccccccc}
\hline Model & System & $f_{1}$ & $f_{2}$ & $f_{3}$ & $f_{4}$ & $f_{5}$ \\
\hline p3 & Abaqus & $61.57(4)$ & $69.10(6)$ & $79.24(7)$ & $83.24(9)$ & $85.99(10)$ \\
offset & & & & & & \\
p3 & Abaqus & $68.52(4)$ & $71.27(5)$ & $76.33(7)$ & $79.11(9)$ & $82.00(10)$ \\
p3 & FEAS & 7.70 & 35.00 & 68.04 & 68.50 & 69.27 \\
p3 & ARSAP & $32.89(2)$ & $68.01(3)$ & $69.70(4)$ & $70.86(6)$ & $75.51(7)$ \\
p3 & RFEM & 4.41 & 32.28 & 68.73 & 70.48 & 71.52 \\
\hline
\end{tabular}

In the list of eigenvalues (see Table 9), we can see a big difference in the first eigenvalue, and in Figures 12-15, we can also see large discrepancies for individual modes of natural vibration. 


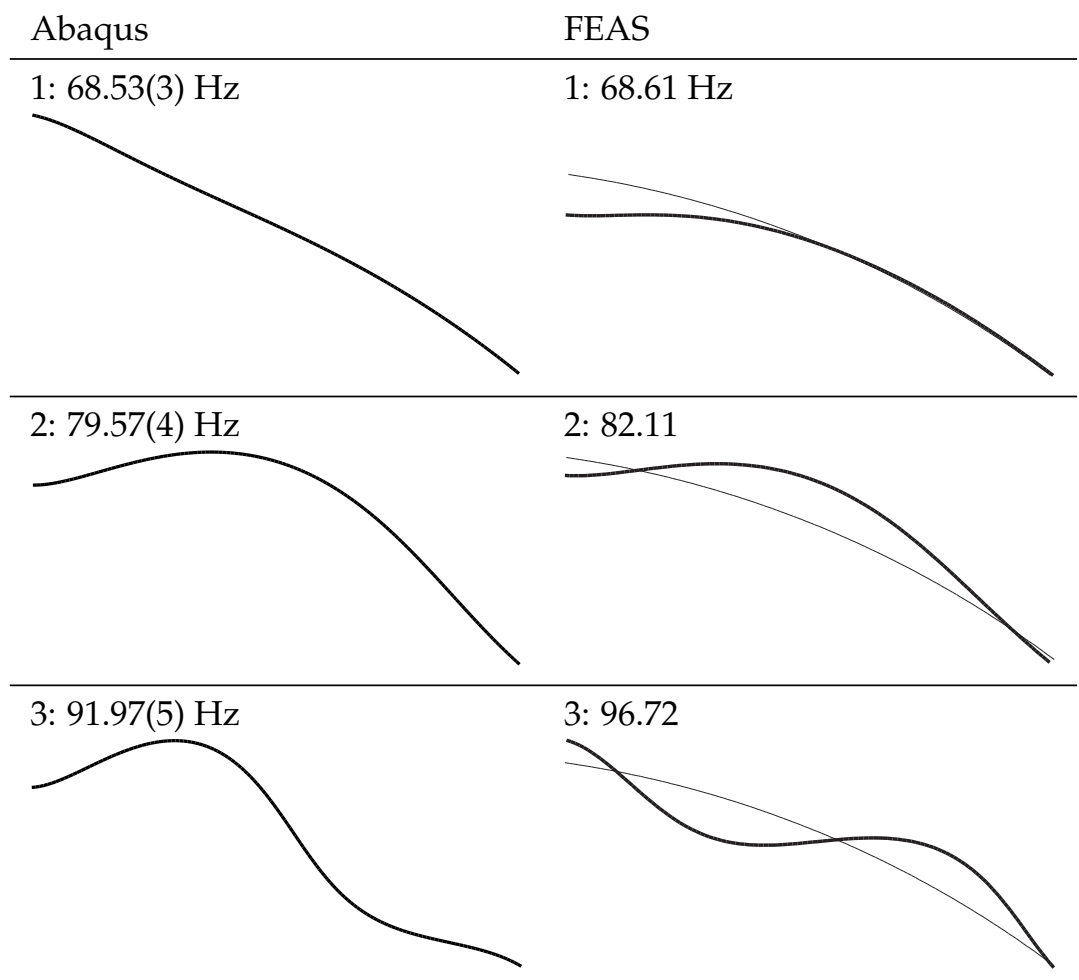

Figure 11. Model p2: frequencies and modes of natural vibration.
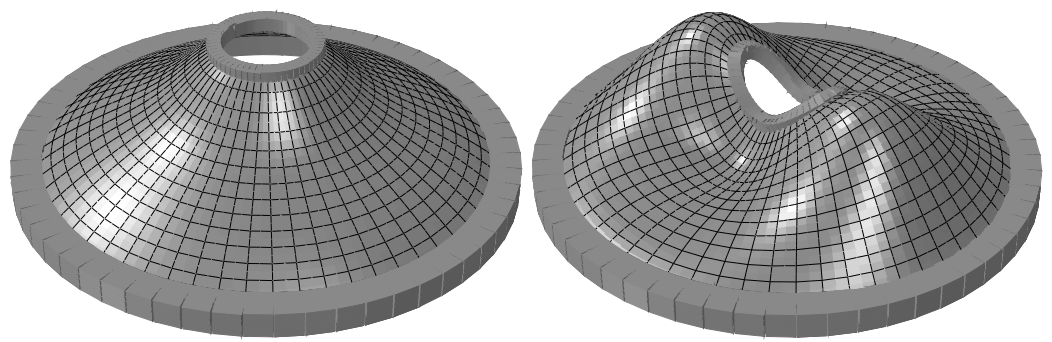

Figure 12. Abaqus, the p3 model: first and third modes of natural vibration.
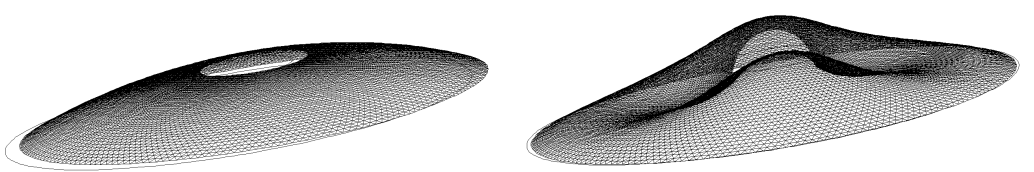

Figure 13. FEAS, the p3 model: first and third modes of natural vibration.
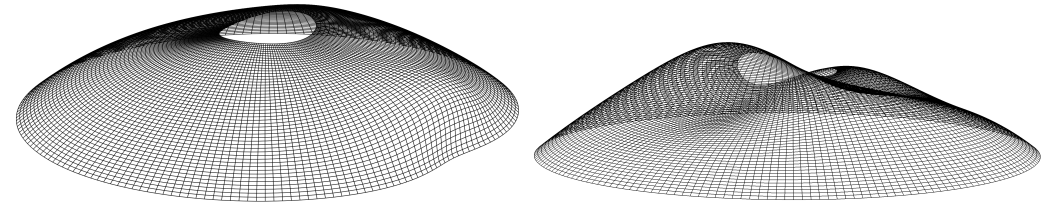

Figure 14. ARSAP, the p3 model: first and third modes of natural vibration.

As part of the numerical tests, we analyzed the p3 offset model in the Abaqus system, assuming different shell and beam finite elements (with linear and square shape functions): S8R + B32, S8R5 + B32, S4R + B31, S4 + B31. We present the results in Table 10. The discrepancies between different $\mathrm{FE}$ types were very slight. 

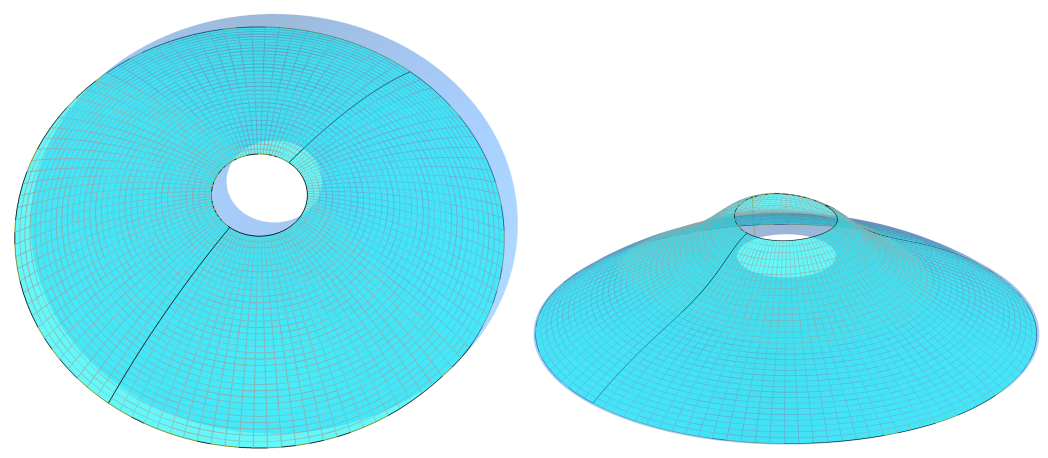

Figure 15. RFEM, the p3 model: first and third modes of natural vibration.

Table 10. List of the first natural frequencies of the shell for the $\mathrm{p} 3$ offset model calculated in the Abaqus system using different FE types $(\mathrm{Hz})$.

\begin{tabular}{cccccc}
\hline Finite Elements & $f_{1}$ & $f_{2}$ & $f_{3}$ & $f_{4}$ & $f_{5}$ \\
\hline S8R+B32 & $61.57(4)$ & $69.10(6)$ & $79.24(7)$ & $83.24(9)$ & $85.99(10)$ \\
S8R5+B32 & $61.24(4)$ & $69.09(6)$ & $79.16(7)$ & $83.26(9)$ & $85.86(10)$ \\
S4R+B31 & $62.17(4)$ & $69.63(6)$ & $79.65(7)$ & $83.63(9)$ & $87.14(10)$ \\
S4+B31 & $61.86(4)$ & $69.56(6)$ & $79.54(7)$ & $83.54(9)$ & $86.86(10)$ \\
\hline
\end{tabular}

In Table 11, the first natural frequencies for the $\mathbf{p} 4$ model are complied.

Table 11. List of the first natural frequencies of the dome for the $\mathbf{p} 4$ model $(\mathrm{Hz})$.

\begin{tabular}{cccccc}
\hline System & $f_{1}$ & $f_{2}$ & $f_{3}$ & $f_{4}$ & $f_{5}$ \\
\hline Abaqus & 56.55 & 57.80 & 57.87 & 59.42 & 69.86 \\
FEAS & 61.46 & 63.40 & 64.25 & 65.08 & 69.76 \\
ARSAP & 57.49 & 58.85 & $60.31(4)$ & $69.87(5)$ & $71.33(6)$ \\
RFEM & 53.31 & 54.47 & $56.6(4)$ & $70.53(5)$ & $71.81(6)$ \\
\hline
\end{tabular}

The selected modes of natural vibration are shown in Figures 16-19.
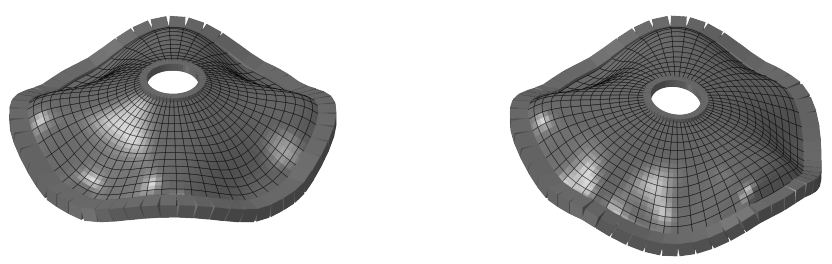

Figure 16. Abaqus, the $\mathrm{p} 4$ model: first and third modes of natural vibration.
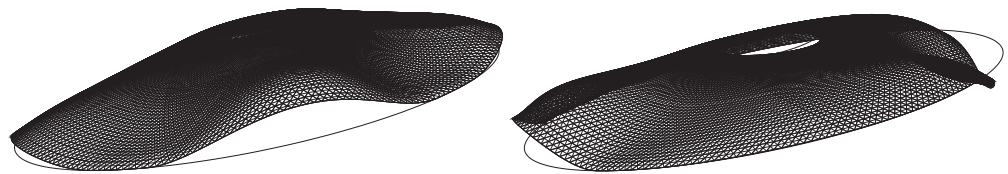

Figure 17. FEAS, the $\mathrm{p} 4$ model: first and third modes of natural vibration. 

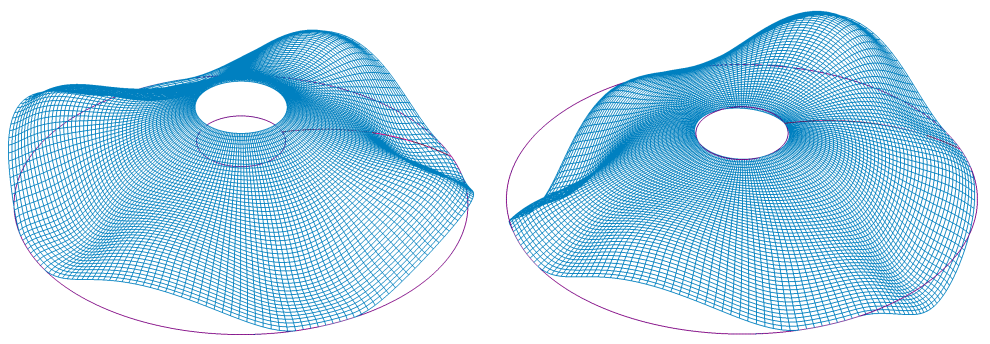

Figure 18. ARSAP, the $\mathbf{p} 4$ model: first and third modes of natural vibration.
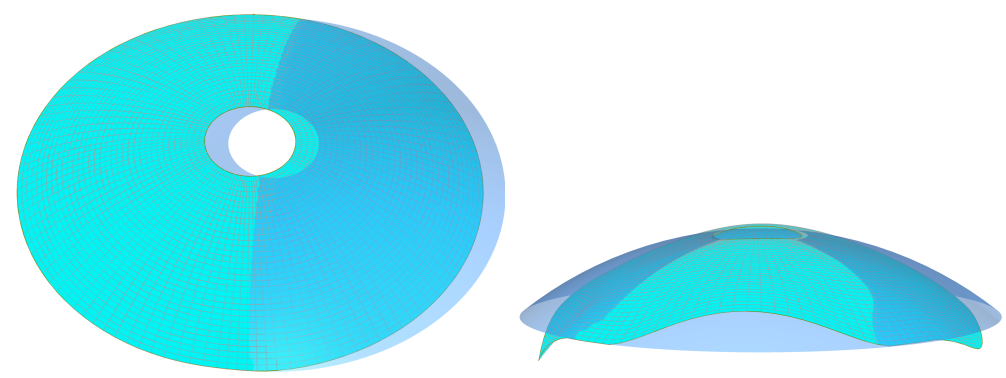

Figure 19. RFEM, the p4 model: first and third modes of natural vibration.

With the use of 3D models, it is possible to determine the forms of natural vibration, which are not only rotationally symmetric, while 2D models are able to capture only these ones. Therefore, comparing the results obtained with different models is more difficult. Table 12 lists the natural frequencies for the individual models to which the first approximately rotationally symmetric modes of free vibration correspond. In the case of the models that were analyzed in at least three systems, the mean, STD, and CoV were also calculated, allowing for the evaluation of the dispersion of the results obtained with the use of different systems. The quotients of the calculated natural frequencies and the reference value (in bold in the table) are given in parentheses, which were assumed as averages for the $\mathbf{p} 1$ model, excluding the erroneous results from the ARSAP system.

Table 12. List of the natural frequencies that correspond to the first rotationally symmetrical form of free vibration.

\begin{tabular}{llllllll}
\hline & $\mathbf{p} 1$ & $\mathbf{p} 2$ & & $\mathbf{p 3}$ & & $\mathbf{p} 4$ & \\
\hline Abaqus & 68.9 & 68.5 & $(0.99)$ & 68.5 & $(0.99)$ & 69.9 & $(1.01)$ \\
FEAS & 69.54 & 68.6 & $(0.99)$ & 68.5 & $(0.99)$ & 69.9 & $(1.01)$ \\
ARSAP & 171.5 & & & 69.7 & $(1.01)$ & 69.9 & $(1.01)$ \\
Dlubal & & & & 68.7 & $(0.99)$ & 70.5 & $(1.02)$ \\
\hline mean & 103.3 & & & 68.8 & $(0.99)$ & 70.0 & $(1.01)$ \\
STD & 48.2 & & & 0.47 & & 0.29 & \\
CoV & 0.47 & & & 0.01 & & 0.004 & \\
ref mean & $\mathbf{6 9 . 2}$ & & & & & & \\
\hline
\end{tabular}

\section{Discussion}

The paper presented a method of verifying the numerical model of a dome with the use of various modeling methods and computational systems. The discussion of the results is divided into two parts concerning the static and dynamic analyses.

\subsection{Results of the Static Analysis}

The vertical displacements obtained from individual models are listed in Tables 4 and 5 . In the following considerations, we assumed that the axisymmetric model $\mathbf{p} \mathbf{1}$ is the reference model for the $\mathbf{p} 2$ and $\mathbf{p} 3$ models because it uses the continuum elements in the state 
of axisymmetry (without the simplifying assumptions), and the analysis was carried out using dense meshes, which ensured the most accurate results.

In the case of self-weight, the differences between the models did not exceed $10 \%$, which can be considered a sufficient accuracy for engineering calculations. However, attention should be paid to the displacement, when the load is applied to the top ring: the differences between the models were surprisingly large here, and the results differed by up to $36 \%$. It can be concluded that both the shell-bar models (p3) and the rotationally symmetric model with ring elements (p2) from the Abaqus system poorly reflected the analyzed dome, more precisely the cooperation between the ring beams and the dome: the omission of the eccentricities significantly reduced the stiffness of the system (see the result for the $\mathbf{p} 3$ offset model). The compatibility between the $\mathbf{p} 3$ models may be misleading: by analyzing only these models, obtained by different computational systems, it is easy to confirm that we obtained a good representation of the structure ( $\mathrm{CoV}$ in the $2 \div 8 \%$ range). Only the verification using the solid axisymmetric model showed the scale of the error. This is a good example of a situation where the verification and validation limited to one model (in this case, the shell-bar one) do not guarantee the correct results.

The analysis of the results for the $\mathbf{p} 4$ model showed the influence of the boundary conditions on the displacement distribution. In the analyzed example, it was greater in the case of a self-weight (difference up to $84 \%$ ) than in the case of a ring beam load (difference up to $10 \%$ ). The dispersion of the results obtained with the use of different computational systems was slightly larger for the self-weight load ( $\mathrm{CoV}$ up to $8 \%$ ) compared to the top ring load (CoV up to $6 \%)$.

\subsection{Results of the Modal Analysis}

The analysis of the dynamic properties of the structure is usually a more difficult task that requires some experience. The paper showed that the verification and validation of the model for static analysis are mostly independent of the model for dynamic analysis. The performed calculations clearly showed that a correct model for the static case does not mean that we will obtain valuable results in the dynamics. Despite similar results in terms of the static case, significantly different natural frequencies were obtained, in particular for the $\mathbf{p} 1$ model. What is more, the solid revolution symmetric model (p1) from the ARSAP program produced erroneous results (differing by 150\% compared to the other models). This should be clearly emphasized as ARSAP is one of the most popular systems used in engineering calculations. On the other hand, the presence of an error in such a popular system for structural designing proves that modal analysis of an axisymmetric model is rarely carried out in practice. This is not surprising, since after analyzing the results for the p3 and p4 models, it can be concluded that the first modes of free vibration were not axisymmetric, so a three-dimensional model must be prepared for dynamic analyses.

The eigenvalue problem, however, is sometimes used as a computational model check in static problems. Consequently, it is possible to find modeling errors that are difficult to detect with static calculations alone. The error of the solution in the modal analysis is difficult to estimate due to the complicated calculation procedures, which again confirms the importance of the verification and validation of the calculations performed with FEM.

In the case of modal analysis, taking into account only the frequencies of free vibration corresponding to axisymmetric eigenmodes and the rejection of the erroneous result from the ARSAP system, the differences between the individual models were insignificant $(1 \div 2 \%$; cf. Table 12). Furthermore, within individual models, the results for different computational systems were characterized by a small spread ( $\mathrm{CoV}$ up to $1 \%$ ).

\section{Conclusions}

The paper showed how important the verification and validation of numerical models is to the stage of engineering calculations. The presented examples showed that, before performing the main part of the static analysis (which includes all load cases, load combinations, code checks, etc.), it is recommended to conduct the calculations with the dead 
weight only and the modal analysis. This approach allows for the detection of gross errors in modeling geometry, connecting individual parts of the model, etc.

In the case of designing complex bar-shell structures, the possibilities of validating numerical models are most often limited. Instead of this, one can use the algorithm shown in this paper, i.e., verify the results of complex models (which are now more and more popular and will probably only gain popularity) with simpler, e.g., $2 \mathrm{D}$, models. In the case of rotationally symmetric models, more precise results in the problem of static analysis were obtained, as the examples from this work showed. It should be emphasized here that even the most popular engineering computational systems can lead to erroneous results, as for the $\mathbf{p} \mathbf{1}$ model in the ARSAP system for the modal analysis. In such a situation, it is worth verifying the model with another system.

Author Contributions: Conceptualization, Z.K.; methodology, Z.K.; software, P.C., S.D. and Z.K.; validation, P.C., S.D. and Z.K.; formal analysis, P.C., S.D. and Z.K.; investigation, P.C., S.D. and Z.K.; resources, Z.K.; data curation, S.D. and Z.K.; writing-original draft preparation, P.C., S.D. and Z.K.; writing-review and editing, P.C., S.D. and Z.K.; visualization, P.C., S.D. and Z.K.; supervision, Z.K.; project administration, Z.K.; funding acquisition, Z.K. All authors have read and agreed to the published version of the manuscript.

Funding: This research received no external funding.

Institutional Review Board Statement: Not applicable.

Informed Consent Statement: Not applicable.

Data Availability Statement: The data presented in this study are available upon request from the authors.

Conflicts of Interest: The authors declare no conflict of interest.

\section{References}

1. Kwasniewski, L.; Bojanowski, C. Principles of verification and validation. J. Struct. Fire Eng. 2015, 6, 29-40. [CrossRef]

2. Roache, P. Verification and Validation in Computational Science and Engineering; Hermosa Publishers: Albuquerque, Mexico, 1998.

3. Perelmuter, A.V.; Slivker, V.I. Numerical Structural Analysis: Methods, Models and Pitfalls; Springer: Berlin/Heidelberg, Germany, 2003; p. 1005. [CrossRef]

4. Kobielak, S.; Zamiar, Z. Oval concrete domes. Arch. Civ. Mech. Eng. 2017, 17, 486-501. [CrossRef]

5. Mazurkiewicz, Z. Thin Elastic Shells: Linear Theory; WUT Publishing House: Warsaw, Poland, 2004. (In Polish)

6. Radwańska, M. Shell Structures. Theoretical Background and Selected Analytical and Numerical Solutions; CUT Publishing House: Kraków, Poland, 2013. (In Polish)

7. Godycki-Ćwirko, T.; Nagrodzka-Godycka, K.; Wojdak, R. Reinforced concrete thin-wall dome after eighty years of operation in a marine climate environment. Struct. Concr. 2016, 17, 710-717. [CrossRef]

8. Sharbaf, A.; Bemanian, M.; Daneshjoo, K.; Shakib, H. Masonry Dome Behavior under Gravity Loads Based on the Support Condition by Considering Variable Curves and Thicknesses. Buildings 2021, 11, 241. [CrossRef]

9. Shen, X.; Zhang, Q.; Lee, D.S.H.; Cai, J.; Feng, J. Static Behavior of a Retractable Suspen-Dome Structure. Symmetry 2021, $13,1105$. [CrossRef]

10. Opatowicz, D.; Radoń, U.; Zabojszcza, P. Assessment of the Effect of Wind Load on the Load Capacity of a Single-Layer Bar Dome. Buildings 2020, 10, 179. [CrossRef]

11. Pantò, B.; Cannizzaro, F.; Caddemi, S.; Caliò, I.; Chácara, C.; Lourenço, P.B. Nonlinear Modeling of Curved Masonry Structures after Seismic Retrofit through FRP Reinforcing. Buildings 2017, 7, 79. [CrossRef]

12. Jasieńko, J.; Raszczuk, K.; Kleszcz, K.; Frąckiewicz, P. Numerical analysis of historical masonry domes: A study of St. Peter's Basilica dome. Structures 2021, 31, 80-86. [CrossRef]

13. Pokorska-Silva, I.; Kadela, M.; Fedorowicz, L. A reliable numerical model for assessing the thermal behavior of a dome building. J. Build. Eng. 2020, 32, 101706. [CrossRef]

14. Pokorska-Silva, I.; Kadela, M.; Małek, M.; Fedorowicz, L. An Assessment of the Thermal Behavior of Envelope Surface Coatings with Different Colors. Polymers 2021, 13, 82. [CrossRef] [PubMed]

15. Czumaj, P.; Dudziak, S.; Kacprzyk, Z. Computational models of reinforced concrete ribbed floor. MATEC Web Conf. 2018, 196, 1-8. [CrossRef]

16. Lewiński, P.P.M.; Dudziak, S. Nonlinear analysis of RC cylindrical tank and subsoil accounting for a low concrete strength. MATEC Web Conf. 2017, 117, 107. [CrossRef] 
17. Lewiński, P.M.; Dudziak, S. Nonlinear interaction analysis of RC cylindrical tank with subsoil by adopting two kinds of constitutive models for ground and structure. AIP Conf. Proc. 2018, 1922, 130007. [CrossRef]

18. Zobel, H.; Zbiciak, A.; Oleszek, R.; Michalczyk, R.; Mossakowski, P. Numerical identification of the dynamic characteristics of a steel-concrete railway bridge. Roads Bridg.-Drog. I Mosty 2014, 13, 275-301. [CrossRef]

19. Cotsovos, D.M.; Zeris, C.A.; Abbas, A.A. Finite Element Modeling of Structural Concrete. In Proceedings of the 2nd International Conference on Computational Methods in Structural Dynamics and Earthquake Engineering 2009, Rodos, Greece, 22-24 June 2009.

20. Autodesk. Robot Structural Analysis Professional. 2021. Available online: https://help.autodesk.com/view/RSAPRO/2021 /PLK/ (accessed on 4 November 2021).

21. Dlubal Software Sp. z o.o. RFEM 5: Calculation of Spatial Structures Using the Finite Element Method. Program Description. 2015. Available online: https://www.dlubal.com/pl/pliki-do-pobrania-i-informacje/dokumenty/instrukcje (accessed on 4 November 2021). (In Polish)

22. Dassault Systèmes. Manual Abaqus 6.14; Dassault Systèmes: Velizy-Villacoublay, France, 2014.

23. Kacprzyk, Z. FEAS—Finite Element Method Structure Analysis System. Comput. Methods Civ. Eng. 1991, 1, 51-65. (In Polish)

24. Jankowska, J.; Jankowski, M. Review of Numerical Methods and Algorithms; WNT: Warsaw, Poland, 1988. (In Polish)

25. Parlett, B.N. The Symmetric Eigenvalue Problem; Prentice-Hall International Series in Computer Science; Prentice-Hall: Hoboken, NJ, USA, 1980.

26. Kacprzyk, Z.; Czumaj, P.; Dudziak, S. Modeling of Building Structures; WUT Publishing House: Warsaw, Poland, 2021. 